\title{
Dynamics of gauge systems and Dirac's conjecture
}

\author{
M. E. V. Costa, H. O. Girotti, and T. J. M. Simões \\ Instituto de Física, Universidade Federal do Rio Grande do Sul, 90000 Porto Alegre, RS, Brasil \\ (Received 15 September 1983; revised manuscript received 28 December 1984)
}

For systems possessing only first-class constraints, we rigorously prove that the secondary constraints act as independent generators of gauge transformations (Dirac's conjecture). The proof essentially consists in demonstrating that the total and the extended Hamiltonian generate the same time evolution for the canonical realization, $f(q, p)$, of the gauge-invariant quantities. We also discuss the alternative realization of these quantities.

\section{INTRODUCTION}

In his work on Hamiltonian dynamics of constrained systems, Dirac ${ }^{1}$ conjectured that all secondary first-class constraints (SFCC's) are independent generators of gauge transformations. If this conjecture holds true, then the dynamics of a system possessing primary $\left\{\phi_{\rho}=0\right.$, $\rho=1,2, \ldots, P\}$ and secondary $\left\{\chi_{\lambda}=0, \lambda=P+1, \ldots\right.$, $P+S \equiv R\}$ first-class constraints should be correctly described by the equations of motion arising from the extended Hamiltonian $H_{E}$,

$$
H_{E}=H+\sum_{\rho=1}^{P} \xi^{\rho} \phi_{\rho}+\sum_{\lambda=P+1}^{R} \xi^{\lambda} \chi_{\lambda},
$$

where the $\xi$ 's are arbitrary Lagrange multipliers and the Hamiltonian $H$ is derived in the manner of Dirac from the Lagrangian $L$ defining the system. ${ }^{1}$

From time to time there have been objections to Dirac's conjecture. $^{2-10}$ All these objections are based on the straightforward observation that, in general, the equations of motion deriving from $H_{E}$ are not strictly equivalent to the corresponding Lagrange equations. For this reason, the total Hamiltonian $\boldsymbol{H}_{T}$,

$$
H_{T}=H+\sum_{\rho=1}^{P} \xi^{\rho} \phi_{\rho}
$$

has been advocated by many authors as the correct timedevelopment generator for constrained systems. ${ }^{5-11}$ In fact, one can recover from the Hamiltonian equations of motion generated by $H_{T}$ the corresponding Lagrangian equations of motion.

In this paper we show that Dirac's conjecture holds true for all gauge systems possessing only first-class constraints (FCC's). By this we mean that

(a) $H_{E}$ and $H_{T}$ may generate different equations of motion for gauge-dependent variables;

(b) nevertheless, $H_{E}$ and $H_{T}$ generate the same equations of motion for the canonical realization, $f(q, p)$, of the gauge-invariant quantities of the system.

In other words, we shall be proving here that $H_{E}$ and $H_{T}$ give rise to the same physics.

In Sec. II we give a general proof of the above state- ments. We also discuss here the alternative realizations of the gauge-invariant quantities. Electrodynamics and the Christ-Lee model ${ }^{12,13}$ are used in Sec. III to exemplify the equivalence of the dynamical pictures generated by $H_{T}$ and $H_{E}$. Some final remarks and the conclusions are contained in Sec. IV.

\section{DYNAMICS \\ OF THE GAUGE-INVARIANT QUANTITIES}

We shall be dealing with systems possessing only FCC's and, therefore, verifying the involution algebra ${ }^{14,15}$

$$
\begin{aligned}
& {\left[\Omega_{a}(q, p), \Omega_{b}(q, p)\right]=\sum_{c=1}^{R} C_{a b}^{c}(q, p) \Omega_{c}(q, p),} \\
& {\left[H(q, p), \Omega_{a}(q, p)\right]=\sum_{b=1}^{R} B_{a}^{b}(q, p) \Omega_{b}(q, p),}
\end{aligned}
$$

where $a, b$, and $c$, run from 1 to $R$ and

$$
\begin{aligned}
& \Omega_{a} \equiv \phi_{a} \approx 0, \quad a=1,2, \ldots, P, \\
& \Omega_{a} \equiv \chi_{a} \approx 0, \quad a=P+1, \ldots, R .
\end{aligned}
$$

The sign of weak equality $(\approx)$ is defined as in Ref. 1 . Furthermore, $C_{a b}^{c}$ and $B_{a}^{b}$ are certain functions of the canonical variables $q \equiv\left(q^{1}, \ldots, q^{N}\right), p \equiv\left(p_{1}, \ldots, p_{N}\right)$. These variables span the phase space $\Gamma$ and $[f, h]$ is the usual Poisson bracket in $\Gamma$. By assumption the constraints are independent and irreducible ${ }^{15}$ in the sense that Eqs. (2.3) define a hypersurface $\Sigma(\Sigma \subset \Gamma)$ of the dimension $2 N-R$. Of course, $R<N$. A quantity $f(q, p)$ is said to be first class if

$$
\left[f(q, p), \Omega_{a}(q, p)\right]=\sum_{b=1}^{R} D_{a}^{b}(q, p) \Omega_{b}(q, p) \approx 0 .
$$

Clearly, (2.2) expresses that $H$ is first class.

We designate by $g(q, p, t)$ the generator of infinitesimal gauge transformations leaving invariant the constraint equations (2.3) and the equations of motion

$$
\begin{aligned}
& \dot{q}^{\rho} \approx \xi^{\rho}, \quad \rho=1,2, \ldots, P, \\
& \dot{q}^{\alpha} \approx\left[q^{\alpha}, H_{T}\right], \quad \alpha=P+1, \ldots, N,
\end{aligned}
$$




$$
\dot{p}_{r} \approx\left[p_{r}, H_{T}\right], \quad r=1,2, \ldots, N,
$$

deriving from $H_{T}$. Here the overdot denotes differentiation with respect to time and the $\dot{q}^{\rho}$ 's are the "unsolved" velocities. ${ }^{16-18}$ In $(2.5 \mathrm{a})$ use was made of the general form of the primary constraints $\phi_{\rho}=p_{\rho}-\eta_{\rho}\left(q^{r}, p_{\alpha}\right){ }^{6,17,18}$ As in Ref. 17, the Lagrange multipliers are supposed to remain untouched by the Poisson bracket operation. An explicit expression for $g(q, p, t)$ in terms of the constraints can be obtained by demanding invariance of (2.3) and (2.5) under the gauge transformation

$$
\begin{aligned}
& \delta q^{r} \approx\left[q^{r}, g\right], \\
& \delta p_{r} \approx\left[p_{r}, g\right] \\
& \delta \xi^{\rho} \approx \delta \dot{q}^{\rho} \equiv \frac{d}{d t}\left[q^{\rho}, g\right] .
\end{aligned}
$$

After some calculations one arrives at

$$
\begin{aligned}
g(q, p, t)=\sum_{\rho=1}^{P} & \frac{d^{K} \epsilon^{\rho}(t)}{d t^{K_{\rho}}} \phi_{\rho}(q, p) \\
& \left.+\sum_{n=0}^{K_{\rho}-1} \frac{d^{n} \epsilon^{\rho}(t)}{d t^{n}} \chi_{\rho, n}(q, p)\right),
\end{aligned}
$$

in agreement with previous results., 5,19 In Eq. (2.7) $\left\{\chi_{\rho, n} \approx 0, n=0,1, \ldots, K_{\rho}-1\right\}$ is the subset of SFCC's originating from the primary first-class constraints (PFCC's) $\phi_{\rho} \approx 0$ through Castellani's algorithm. ${ }^{6,20}$ Moreover, the $\epsilon^{\rho}(t)$ 's are infinitesimal independent parameters related to the $\delta \xi^{\rho}$ 's as

$$
\begin{aligned}
& \delta \xi^{\rho}= \frac{d^{K_{\rho}+1} \epsilon^{\rho(t)}}{d t^{K_{\rho}+1}} \\
&+\sum_{\sigma=1}^{P}\left[\frac{d^{K_{\sigma} \epsilon^{\sigma}(t)}}{d t^{K_{\sigma}}}\left[q^{\rho}, \chi_{\sigma, K_{\sigma}-1}\right]\right. \\
&+\sum_{n=0}^{K_{\sigma}-1} \frac{d^{n} \epsilon^{\sigma}(t)}{d t^{n}}\left[q^{\rho},\left[\chi_{\sigma, n}, H_{T}\right]\right. \\
&\left.\left.+\chi_{\sigma, n-1}\right]\right] .
\end{aligned}
$$

In this last expression one is to take $\chi_{\sigma,-1} \equiv 0$. We remark that all FCC's are included in $g(q, p, t),{ }^{6}$ since we are leaving out of consideration systems such as those proposed by Allcock ${ }^{2}$ and Cawley, ${ }^{3}$ for which some of the SFCC's arise as powers $\chi^{m}$ with $m>1$. For the justmentioned models the apparent failure of Dirac's conjecture results from an improper linearization of the functional forms of the SFCC's. ${ }^{21}$

The physically meaningful quantities in a gauge system are those functions $f(q, p)$ being invariant under the gauge transformations (2.6), i.e.,

$$
[f(q, p), g(q, p, t)] \approx 0 .
$$

Now (2.9) calls for

$$
\begin{aligned}
& \frac{d^{K} e^{\rho}(t)}{d t^{K}}\left[f(q, p), \phi_{\rho}(q, p)\right] \\
& +\sum_{n=0}^{K_{\rho}-1} \frac{d^{n} \epsilon^{\rho}(t)}{d t^{n}}\left[f(q, p), \chi_{\rho, n}(q, p)\right] \approx 0
\end{aligned}
$$

for all $\rho$, as seen from (2.7). The terms in (2.10), each one of which contains a time derivative of $\epsilon^{\rho}(t)$ of different order, cannot compensate among themselves since no time derivatives of $q$ and/or $p$ appear in the argument of $f$. Then, for (2.10) to hold we must have

$$
\left[f(q, p), \Omega_{a}(q, p)\right] \approx 0, \quad a=1,2, \ldots, R .
$$

From (2.11) and (2.4) follows that $f(q, p)$ is first class. This is a necessary and sufficient condition for $f(q, p)$ to be gauge invariant. But (2.11) is also a necessary and sufficient condition for $f(q, p)$ to be invariant under the $e x$ tended infinitesimal transformations

$$
\begin{aligned}
& \delta q^{r} \approx\left[q^{r}, G\right], \\
& \delta p_{r} \approx\left[p_{r}, G\right],
\end{aligned}
$$

generated by

$$
G(q, p, t)=\sum_{a=1}^{R} \tau^{a}(t) \Omega_{a}(q, p),
$$

where the $\tau^{a}(t)$ 's are arbitrary independent parameters. Hence, for any given gauge system, $g$ and $G$ define the same set of canonical gauge-invariant functions $f(q, p)$.

Now, by construction, $G$ leaves invariant the equations of motion

$$
\begin{aligned}
& \dot{q}^{r} \approx\left[q^{r}, H_{E}\right], \\
& \dot{p}_{r} \approx\left[p_{r}, H_{E}\right],
\end{aligned}
$$

deriving from the extended Hamiltonian [see (1.1) and (2.3)]

$$
H_{E}=H+\sum_{a=1}^{R} \xi^{a} \Omega_{a},
$$

provided Eqs. (2.12) are supplemented with ${ }^{14}$

$$
\delta \xi^{a}=\dot{\tau}^{a}-\sum_{b=1}^{R} B_{b}^{a} \tau^{b}+\sum_{b, c=1}^{R} C_{b c}^{c} \tau^{b} \xi^{c} .
$$

The sets of equations of motion (2.5) and (2.14) are different, since $H_{T}$ and $H_{E}$ differ by a linear combination of SFCC's. Furthermore, the Lagrangian equations of motion can be recovered from (2.5) but not, in general, from (2.14). This fact has led many authors ${ }^{5-10}$ to reject the extended Hamiltonian as the generator of a reliable dynamical picture. However, from (1.2), (2.15), and (2.11) one finds

$$
\dot{f} \approx\left[f, H_{T}\right] \approx\left[f, H_{E}\right],
$$

which explicitly shows that $H_{T}$ and $H_{E}$ generate the same time evolution for the canonical gauge-invariant functions $f(q, p)$. To phrase it differently: $H_{E}$ and $H_{T}$ are physically equivalent and, as a consequence, Dirac's conjecture holds true for the systems under analysis.

Although $H_{E}$ and $H_{T}$ are physically equivalent, as we 
just proved, they generate different equations of motion for the gauge-dependent variables. We turn now to analyzing some consequences of this difference. Notice that one may use the equations of motion deriving from $H_{T}$ to recast a gauge-invariant function $f(q, p)$ into the form $f_{T}\left(q^{r}, \dot{q}^{r}, p_{r}, \dot{p}_{r}, \xi^{\rho}\right)$. Analogously, one can obtain $f_{E}\left(q^{r}, \dot{q}^{r}, p_{r}, \dot{p}_{r}, \xi^{a}\right)$ from $f(q, p)$ by using the equations of motion deriving from $H_{E}$. By construction, $f_{T}$ is invariant under (2.6); indeed, $f(q, p)$ satisfies (2.9) and $g$ was built so as to leave invariant the equations of motion deriving from $H_{T}$. On the other hand, and also by construction, $f_{E}$ is invariant under (2.12) and (2.16). However, $f_{T}$ is not invariant under the set of extended transformations (2.12) and (2.16) generated by $G$. We emphasize that $f_{T}$ and $f_{E}$ depend upon different arguments. In fact, the Lagrange multipliers $\xi^{a}, a=P+1, \ldots, R$, only appear in the argument of $f_{E}$ and, therefore, it makes no sense whatsoever to compare $f_{T}$ with $f_{E}$.

We, then, conclude that a given gauge-invariant function, with canonical form $f(q, p)$, admits alternative realizations, such as $f_{T}$ and $f_{E}$, which are faithful only within the formalism $\left(H_{T}\right.$ or $\left.H_{E}\right)$ where they originated. The existence of these alternative "formalism-dependent" realizations for a gauge-invariant quantity has been a source of confusion in the literature. ${ }^{6-10}$ We shall come back to this point in the next section.

\section{EXAMPLES}

\section{A. Electrodynamics}

From the Lagrangian density ${ }^{22}$

$$
\mathscr{L}=-\frac{1}{4} F_{\mu \nu} F^{\mu v}+\bar{\psi}\left[i \gamma^{\mu}\left(\partial_{\mu}-i e A_{\mu}\right)-m\right] \psi,
$$

where $F^{\mu v}=\partial^{\mu} A^{v}-\partial^{v} A^{\mu}$, one finds the canonical conjugate momenta

$$
\begin{aligned}
& \pi_{0}=0, \\
& \pi_{j}=F^{0 j}, \\
& \pi_{\psi}=i \bar{\psi} \gamma^{0},
\end{aligned}
$$

and the Hamiltonian

$$
\begin{gathered}
H=\int d^{3} x\left[\frac{1}{2} \pi_{j} \pi_{j}-A^{0}\left(\partial^{j} \pi_{j}-i e \pi_{\psi} \psi\right)+\frac{1}{4} F^{i j} F^{i j}\right. \\
+\frac{1}{2} \pi_{\psi} \gamma^{0} \gamma^{k}\left(\partial^{k} \psi\right)-\frac{1}{2}\left(\partial^{k} \pi_{\psi}\right) \gamma^{0} \gamma^{k} \psi \\
\left.-i e \pi_{\psi} \gamma^{0} \gamma^{k} \psi A^{k}-i m \pi_{\psi} \gamma^{0} \psi\right]
\end{gathered}
$$

The PFCC and the SFCC are, respectively,

$$
\begin{aligned}
& \Omega_{1} \equiv \pi_{0} \approx 0, \\
& \Omega_{2} \equiv \partial^{j} \pi_{j}-i e \pi_{\psi} \psi \approx 0 .
\end{aligned}
$$

Theses constraints and the Hamiltonian (3.3) obey the involution algebra (2.1) and (2.2) with

$$
\begin{aligned}
& C_{a b}^{c}=0, \quad a, b, c,=1,2 ; \\
& B_{1}^{1}=B_{2}^{1}=B_{2}^{2}=0, \quad B_{1}^{2}=-1 .
\end{aligned}
$$

Then, for electrodynamics (2.7), (2.6), and (2.8) reduce, respectively, to ${ }^{23}$

$$
\begin{aligned}
& g=\int d^{3} x\left\{\dot{\epsilon}(\mathbf{x}) \pi_{0}(\mathbf{x})\right. \\
& \left.-\epsilon(\mathbf{x})\left[\partial^{j} \pi_{j}(\mathbf{x})-i e \pi_{\psi}(\mathbf{x}) \psi(\mathbf{x})\right]\right\}, \\
& \delta A^{\mu}(\mathbf{x})=\partial^{\mu} \epsilon(\mathbf{x}), \\
& \delta \psi(\mathbf{x})=i e \epsilon(\mathbf{x}) \psi(\mathbf{x}), \\
& \delta \pi_{\mu}(\mathbf{x})=0, \\
& \delta \pi_{\psi}(\mathbf{x})=-i e \epsilon(\mathbf{x}) \pi_{\psi}(\mathbf{x}),
\end{aligned}
$$

and

$$
\delta \xi^{1}(\mathbf{x})=\partial^{0} \partial^{0} \epsilon(\mathbf{x}),
$$

where we have omitted the time label in the fields argument. One can verify the invariance under (3.7) of the equations of motion

$\partial^{0} A^{0}=\xi^{1}$,

$\partial^{0} A^{j}=\pi_{j}+\partial^{j} A^{0}$,

$\partial^{0} \psi=\gamma^{0} \gamma^{k} \partial^{k} \psi-\left(i e \gamma^{0} \gamma^{k} A^{k}+i m \gamma^{0}-i e A^{0}\right) \psi$,

$\partial^{0} \pi_{0}=0$,

$\partial^{0} \pi_{j}=\partial^{i} F^{i j}+i e \pi_{\psi} \gamma^{0} \gamma^{j} \psi$

$\partial^{0} \pi_{\psi}=\partial^{k} \pi_{\psi} \gamma^{0} \gamma^{k}+\pi_{\psi}\left(i e \gamma^{0} \gamma^{k} A^{k}+i m \gamma^{0}-i e A^{0}\right)$,

deriving from the total Hamiltonian,

$$
H_{T}=H+\int d^{3} x \xi^{1}(\mathbf{x}) \pi_{0}(\mathbf{x}) .
$$

The sign of strong equality in (3.7) and (3.8) indicates that these equations have already been written on the constraint surface $\Sigma$.

We next recognize $F^{i j}, \pi_{j}, \pi_{\psi} \psi$, and $\pi_{\psi} \gamma^{0} \gamma^{j} \psi$ as the canonical forms of the basic gauge-invariant quantities of electrodynamics. One can easily check that all these functionals are indeed first class. Thus, $F^{i j}, \pi_{j}, \pi_{\psi} \psi$, and $\pi_{\psi} \gamma^{0} \gamma^{j} \psi$ are also invariant under the extended infinitesimal transformations [see Eqs. (2.12) and (2.16)]

$$
\begin{aligned}
& \delta A^{0}(\mathbf{x})=\tau^{1}(\mathbf{x}), \\
& \delta A^{j}(\mathbf{x})=-\partial^{j} \tau^{2}(\mathbf{x}), \\
& \delta \psi(\mathbf{x})=-i e \tau^{2}(\mathbf{x}) \psi(\mathbf{x}), \\
& \delta \pi_{0}(\mathbf{x})=0 \\
& \delta \pi_{j}(\mathbf{x})=0 \\
& \delta \pi_{\psi}(\mathbf{x})=i e \tau^{2}(\mathbf{x}) \pi_{\psi}(\mathbf{x}), \\
& \delta \xi^{1}(\mathbf{x})=\partial^{0} \tau^{1}(\mathbf{x}), \\
& \delta \xi^{2}(\mathbf{x})=\partial^{0} \tau^{2}(\mathbf{x})+\tau^{1}(\mathbf{x}),
\end{aligned}
$$

generated by

$$
\begin{aligned}
G=\int d^{3} x & \left\{\tau^{1}(\mathbf{x}) \pi_{0}(\mathbf{x})\right. \\
& \left.+\tau^{2}(\mathbf{x})\left[\partial^{j} \pi_{j}(\mathbf{x})-i e \pi_{\psi}(\mathbf{x}) \psi(\mathbf{x})\right]\right\},
\end{aligned}
$$

where $\tau^{1}$ and $\tau^{2}$ are arbitrary infinitesimal parameters. As we asserted in Sec. II, the transformation (3.10) leaves invariant the equations of motion 


$$
\begin{aligned}
& \partial^{0} A^{0}=\xi^{1}, \\
& \partial^{0} A^{j}=\pi_{j}+\partial^{j} A^{0}-\partial^{j} \xi^{2}, \\
& \partial^{0} \psi=\gamma^{0} \gamma^{k} \partial^{k} \psi-\left(i e \gamma^{0} \gamma^{k} A^{k}+i m \gamma^{0}-i e A^{0}+i e \xi^{2}\right) \psi, \\
& \partial^{0} \pi_{0}=0, \\
& \partial^{0} \pi_{j}=\partial^{i} F^{i j}+i e \pi_{\psi} \gamma^{0} \gamma^{j} \psi, \\
& \partial^{0} \pi_{\psi}=\partial^{k} \pi_{\psi} \gamma^{0} \gamma^{k}+\pi_{\psi}\left(i e \gamma^{0} \gamma^{k} A^{k}+i m \gamma^{0}-i e A^{0}+i e \xi^{2}\right),
\end{aligned}
$$

arising from the extended Hamiltonian

$$
\begin{aligned}
H_{E}=H+\int d^{3} x\left\{\xi^{1}(\mathbf{x}) \pi_{0}(\mathbf{x})\right. & \\
& \left.+\xi^{2}(\mathbf{x})\left[\partial^{j} \pi_{j}(\mathbf{x})-i e \pi_{\psi}(\mathbf{x}) \psi(\mathbf{x})\right]\right\} .
\end{aligned}
$$

Here $\xi^{1}$ and $\xi^{2}$ are arbitrary Lagrange multipliers.

As a matter of fact, the sets of equations of motion (3.8) and (3.12) are different. However, irrespective of whether one starts from (3.8) or (3.12) one arrives at the Maxwell equations

$$
\begin{aligned}
& \partial^{0} F^{i j}=\partial^{i} \pi_{j}-\partial^{j} \pi_{i}, \\
& \partial^{0} \pi_{j}=\partial^{i} F^{i j}+i e \pi_{\psi} \gamma^{0} \gamma^{j} \psi,
\end{aligned}
$$

and the local conservation law

$$
\partial^{0}\left(\pi_{\psi} \psi\right)=\partial^{j}\left(\pi_{\psi} \gamma^{0} \gamma^{j} \psi\right)
$$

Therefore, $H_{T}$ and $H_{E}$ generate the same time evolution for the gauge-invariant quantities, as required by (2.17).

We discuss now the alternative formalism-dependent realizations of the electric field $\left(-\pi_{j}\right)$. From (3.8b) one obtains

$$
\pi_{j}=F^{0 j}
$$

Hence, $F^{0 j}$ is a faithful realization of $\pi_{j}$ within the formalism of the total Hamiltonian. We can check that $F^{0 j}$ is invariant under $g$ but not under $G$. On the other hand, the formalism of the extended Hamiltonian provides the equally faithful realization for $\pi_{j}$ [see Eq. (3.12b)]

$$
\pi_{j}=F^{0 j}+\partial^{j} \xi^{2}
$$

which is invariant under (3.10). One should not be puzzled by the fact that (3.18) does not coincide with (3.17) or, what amounts to the same thing, with the Lagrangian definition of $\pi_{j}$ in (3.2b). Actually, in the Hamiltonian formulation the momenta are connected with the coordinates and the time "only through the medium of the equations of motion themselves and not by any a priori defining relationship" (see Ref. 24). Moreover, trying to force (3.17) into the formalism of the extended Hamiltonian may lead, for some gauges, to bizarre results. ${ }^{10}$

\section{B. The Christ-Lee model}

The Christ-Lee model ${ }^{12,13}$ is described by the singular Lagrangian

$$
L=\frac{1}{2}\left[\dot{r}^{2}+r^{2}(\dot{\theta}-z)^{2}\right]-V(r),
$$

which yields the momenta

$$
\begin{aligned}
& p_{r}=\dot{r}, \\
& p_{\theta}=r^{2}(\dot{\theta}-z), \\
& p_{z}=0,
\end{aligned}
$$

and the Hamiltonian

$$
H=\frac{1}{2} p_{r}^{2}+\frac{1}{2 r^{2}} p_{\theta}^{2}+z p_{\theta}+V(r) .
$$

Here, $r$ and $\theta$ are plane polar coordinates, $z$ is another generalized coordinate, and $V(r)$ is a potential bounded from below. By demanding persistence in time of the primary constraint

$$
\Omega_{1} \equiv p_{z} \approx 0,
$$

one finds the secondary constraint

$$
\Omega_{2} \equiv p_{\theta} \approx 0 \text {. }
$$

It is easy to check that there are no further constraints in the theory. The constraints and the Hamiltonian (3.21) are first-class quantities since they obey the involution algebra (2.1) and (2.2) with $C_{a b}^{c}=0, a, b, c=1,2 ; B_{1}^{1}=B_{2}^{1}$ $=B_{2}^{2}=0, B_{1}^{2}=1$.

For the Christ-Lee model (2.6), (2.8), and (2.7) reduce, respectively, to

$$
\begin{aligned}
& \delta r=0, \\
& \delta \theta=\epsilon(t), \\
& \delta z=\dot{\epsilon}(t), \\
& \delta p_{r}=\delta p_{\theta}=\delta p_{z}=0, \\
& \delta \xi^{1}(t)=\ddot{\epsilon}(t),
\end{aligned}
$$

and

$$
g=\dot{\epsilon}(t) p_{z}+\epsilon(t) p_{\theta} .
$$

The equations of motion

$$
\begin{aligned}
& \dot{r}=p_{r}, \\
& \dot{\theta}=z, \\
& \dot{z}=\xi^{1}, \\
& \dot{p}_{r}=-\frac{d V}{d r}, \\
& \dot{p}_{\theta}=0, \\
& \dot{p}_{z}=0,
\end{aligned}
$$

arising from the total Hamiltonian

$$
H_{T}=H+\xi^{1} p_{z}
$$

are invariant under (3.24). Equations (3.26) have the same content as the Lagrange equations deriving from (3.19). The transformations (3.24a), (3.24b), and (3.24c) also leave invariant the Lagrangian (3.19).

The basic canonical gauge-invariant functions $f(q, p)$ for this model are $r, p_{r}, p_{z}$, and $p_{\theta}$. They are all first class and, therefore, invariant under the transformations generated by 


$$
G=\tau^{1}(t) p_{z}+\tau^{2}(t) p_{\theta},
$$

where, again, $\tau^{1}$ and $\tau^{2}$ are arbitrary infinitesimal parameters. Furthermore, the equations of motion

$$
\begin{aligned}
& \dot{r}=p_{r}, \\
& \dot{\theta}=z+\xi^{2}, \\
& \dot{z}=\xi^{1}, \\
& \dot{p}_{r}=-\frac{d V}{d r}, \\
& \dot{p}_{\theta}=0, \\
& \dot{p}_{z}=0,
\end{aligned}
$$

deriving from the extended Hamiltonian

$$
H_{E}=H+\xi^{1}(t) p_{z}+\xi^{2}(t) p_{\theta}
$$

are also invariant under the transformations generated by $G$, i.e.,

$$
\begin{aligned}
& \delta r=0, \\
& \delta \theta=\tau^{2}(t), \\
& \delta z=\tau^{1}(t), \\
& \delta p_{r}=\delta p_{\theta}=\delta p_{z}=0,
\end{aligned}
$$

provided the Lagrange multipliers $\xi^{1}$ and $\xi^{2}$ transform, correspondingly, as [see (2.16)]

$$
\begin{aligned}
& \delta \xi^{1}(t)=\dot{\tau}^{1}(t), \\
& \delta \xi^{2}(t)=\dot{\tau}^{2}(t)-\tau^{1}(t) .
\end{aligned}
$$

The dissimilarities between the sets of equations of motion (3.26) and (3.29) should be noticed. For instance, according to (3.26) we can take either $z$ or $\theta$, but not both, arbitrary, while from (3.29) one concludes that $\theta$ and $z$ are arbitrary functions of time. In other words, all solutions of (3.26) are also solutions of (3.29) but the converse is not true. Nevertheless, both sets of equations yield the same time evolution for the gauge-invariant functions $f(q, p)$ as seen from

$$
\begin{aligned}
& \dot{r}=p_{r}, \\
& \dot{p}_{r}=-\frac{d V}{d r}, \\
& \dot{p}_{z}=0, \\
& \dot{p}_{\theta}=0 .
\end{aligned}
$$

As it happens in electrodynamics, one can build alternative formalism-dependent realizations for the gaugeinvariant functions $f(q, p)$ of the Christ-Lee model. In particular, from (3.23) and (3.26b) one obtains

$$
p_{\theta}=0=r^{2}(\dot{\theta}-z) \text {. }
$$

Hence, $r^{2}(\dot{\theta}-z)$ is a faithful realization of the gaugeinvariant quantity $p_{\theta}$ within the formalism of the total Hamiltonian. One can readily check that $r^{2}(\dot{\theta}-z)$ is invariant under (3.24) but not under (3.31). On the other hand, from (3.23) and (3.29b) one finds

$$
p_{\theta}=0=r^{2}\left(\dot{\theta}-z-\xi^{2}\right) \text {. }
$$

Therefore, the quantity $r^{2}\left(\dot{\theta}-z-\xi^{2}\right)$, which is invariant under (3.31), is a faithful realization of $p_{\theta}$ within the formalism of the extended Hamiltonian. We emphasize again that it makes no sense whatsoever to use the Lagrangian definition of $p_{\theta}(3.20 \mathrm{~b})$ in connection with the equations of motion generated by the extended Hamiltonian.

\section{FINAL REMARKS}

The main feature of a gauge system whose dynamics is described by $H_{T}$ is that the set of equations of motion (2.5) do not possess a unique solution, not even after imposing the initial conditions. ${ }^{1,14-16}$ For each set of Lagrange multipliers $\xi^{\rho}$ one has a different trajectory on the constraint surface $\Sigma$. At any time $t>t_{0}$, where $t_{0}$ is the initial time, the physical state of the system is specified by any point on $\Sigma$ belonging to a certain equivalence class $C_{T}(t)$. The points within each class are connected by the transformations generated by $g$ in (2.7).

When one uses $H_{E}$ instead of $H_{T}$ the gauge freedom is augmented. Actually, the trajectories on $\Sigma$ are now parametrized by the larger set of Lagrange multipliers $\xi^{a}$. The constraint surface divides out again into equivalence classes $C_{E}(t)$; the points within each class now being connected by the transformations generated by $G$ in (2.13). 1,14

Thus, irrespective of whether one works with $H_{T}$ or $H_{E}$, the phase-space image of a physical state of a gauge system is an equivalence class $\left(C_{T}\right.$ or $C_{E}$ ) and not an individual point on $\Sigma$; the dynamics being represented by the motion in time of this equivalence class. As a corroboration of the physical equivalence of $H_{T}$ and $H_{E}$, we shall next demonstrate that $C_{E}(t)=C_{T}(t)$. For this purpose, we start by recasting (2.13) into the form

$$
G(q, p, t)=\sum_{\rho=1}^{P}\left[\tau^{\rho}(t) \phi_{\rho}(q, p)+\sum_{n=0}^{K_{\rho}-1} \tau_{n}^{\rho}(t) \chi_{\rho, n}(q, p)\right]
$$

where the $\tau^{\rho}(t)$ 's and the $\tau_{n}^{\rho}(t)$ 's are all independent parameters. That $C_{T}(t) \subset C_{E}(t)$ follows trivially from (2.7) and (4.1): given the $\epsilon^{\rho}(t)$ 's, one can always choose $\tau^{\rho}(t)=\epsilon^{\rho}(t), \tau_{n}^{\rho}(t)=d^{n} \epsilon^{\rho}(t) / d t^{n}$. The converse, $C_{E}(t)$ $\subset C_{T}(t)$, is also true because, for any instant of time $t$, one can always find a function $\epsilon^{\rho}(t)$ such that $\epsilon^{\rho}(t)=\tau^{\rho}(t)$ and $d^{n} \epsilon^{\rho}(t) / d t^{n}=\tau_{n}^{\rho}(t)$. Thus, $C_{T}(t)=C_{E}(t)$. One can check that the models in Sec. III verify this property. 
${ }^{1}$ P. A. M. Dirac, Lectures on Quantum Mechanics (Yeshiva University, New York, 1964).

${ }^{2}$ G. R. Allcock, Philos. Trans. R. Soc. London A279, 487 (1974).

${ }^{3}$ R. Cawley, Phys. Rev. Lett. 42, 413 (1979); Phys. Rev. D 21, 2988 (1980).

${ }^{4}$ A. Frenkel, Phys. Rev. D 21, 2986 (1980).

${ }^{5}$ N. Mukunda, Phys. Scr. 21, 783 (1980).

${ }^{6}$ L. Castellani, Ann. Phys. (N.Y.) 143, 357 (1982).

${ }^{7}$ R. Sugano, Prog. Theor. Phys. 68, 1377 (1982).

${ }^{8}$ R. Sugano and H. Kamo, Prog. Theor. Phys. 67, 1966 (1982).

${ }^{9}$ R. Sugano and T. Kimura, Prog. Theor. Phys. 69, 252 (1983); 69, 1256 (1983).

${ }^{10}$ R. Sugano and T. Kimura, J. Phys. A 16, 4417 (1983).

${ }^{11}$ N. H. Christ, A. H. Guth, and E. J. Weinberg, Nucl. Phys. B114, 61 (1976).

${ }^{12}$ N. H. Christ and T. D. Lee, Phys. Rev. D 22, 939 (1980).

${ }^{13}$ M. E. V. Costa and H. O. Girotti, Phys. Rev. D 24, 3323 (1981).

${ }^{14}$ E. S. Fradkin and G. A. Vilkovisky, Report No. CERN TH. 1232, 1977 (unpublished).

${ }^{15}$ L. Faddeev, Teor. Mat. Fiz. 1, 3 (1969) [Theor. Math. Phys. 1, 1 (1970)].

${ }^{16}$ E. C. G. Sudarshan and N. Mukunda, Classical Dynamics: $A$ Modern Perspective (Wiley, New York, 1974).

${ }^{17}$ N. Mukunda, Ann. Phys. (N.Y.) 99, 408 (1976).

${ }^{18}$ P. Senjanovic, Ann. Phys. (N.Y.) 100, 227 (1976).

${ }^{19}$ J. L. Anderson and P. G. Bergmann, Phys. Rev. 83, 1018
(1951).

${ }^{20} \mathrm{We}$ notice that the SFCC's arising through the algorithm of Castellani (Ref. 6) are, in the general case, linear combinations of the PFCC's and SFCC's detected via Dirac's algorithm (Ref. 1).

${ }^{21}$ R. Di Stefano, Phys. Rev. D 27, 1752 (1983).

${ }^{22}$ Our metric convention is $g_{00}=-g_{11}=-g_{22}=-g_{33}$ $=+1, g_{\mu \nu}=0$ if $\mu \neq v$.

${ }^{23}$ The Poisson bracket for a field theory including fermions is defined as

$[u, v]=\int d^{3} x\left(\frac{\delta u}{\delta A^{\mu}(\mathbf{x})} \frac{\delta v}{\delta \pi_{\mu}(\mathbf{x})}-(-1)^{n^{n}{ }^{n} v} \frac{\delta v}{\delta A^{\mu}(\mathbf{x})} \frac{\delta u}{\delta \pi_{\mu}(\mathbf{x})}\right)$

$$
+\int d^{3} x\left(\frac{u \overleftarrow{\delta}}{\delta \psi(\mathbf{x})} \frac{\vec{\delta} v}{\delta \pi_{\psi}(\mathbf{x})}-(-1)^{n_{u}{ }^{n} v} \frac{v \overleftarrow{\delta}}{\delta \psi(\mathbf{x})} \frac{\vec{\delta} u}{\delta \pi_{\psi}(\mathbf{x})}\right)
$$

where $u$ and $v$ are arbitrary but regular functions of the phase-space variables, $\vec{\delta} / \delta \psi\left(\overleftarrow{\delta} / \delta \pi_{\psi}\right)$ denotes the left (right) functional derivative, and $n_{u}$ equals 0 or 1 depending on whether $u$ is boson or fermion.

${ }^{24}$ H. Goldstein, Classical Mechanics (Addison-Wesley, Reading, Mass., 1959), p. 227. 